\title{
Effects of scopolamine on smell discrimination in the rat
}

\author{
SANDRA MOLLENAUER, ROD PLOTNIK, and N. JAY BEAN \\ San Diego State University, San Diego, California 92182
}

\begin{abstract}
Earlier work suggested that scopolamine might cause a blockade of olfactory perception. Rats were trained to perform a smell-discrimination and head-poke response for food reinforcement. Following treatment with saline or scopolamine (SCO), rats were retested on these two tasks as well as on passive avoidance. Scopolamine significantly impaired passive avoidance and head-poke responding. Scopolamine also caused a delay in the onset of discrimination performance. There was a significant disruption of smell discrimination on early trials, during which SCO rats failed to execute choices, whether correct or incorrect. However, when SCO rats began to execute choices, their choices were correct. They showed a significant improvement over trials and maintained near-perfect discrimination on the second half of the test. The results of the smell-discrimination test indicate that scopolamine did not cause a complete blockade of olfactory perception. The results of the head-poke test suggest that scopolamine might increase vibrissae sensitivity.
\end{abstract}

In previous research, the anticholinergic drug, scopolamine, was shown to reduce fear responses of rats to a cat (Plotnik, Mollenauer, \& Snyder, 1974). Removal of the rats' olfactory bulbs had a similar effect in reducing fear responses to a cat (Mollenauer, Plotnik, \& Snyder, 1974). Taken together, these findings suggested that scopolamine might be acting on the olfactory system, that is, affecting fear responses indirectly by blocking olfactory perception. Preliminary research with the ether-avoidance procedure described by Spector and Hull (1972) also suggested that scopolamine blocked olfactory perception. In contrast to rats treated with saline, rats treated with scopolamine failed to make the stereotyped head-turning response to an ether-soaked cotton ball.

The present research explored more systematically the possibility that scopolamine caused a general blockade of olfactory perception. Rats were first trained to perform a smell discrimination for food reinforcement. They were then tested for discrimination performance following injections of physiological saline or the dose of scopolamine that had been established in previous research. Two weeks later, the same discrimination test was repeated under the opposite drug conditions and was followed immediately by a second task, head-poke, known to be sensitive to scopolamine. It had been found in pilot work that scopolamine, but not methyl scopolamine, blocked a head-poke discrimination response. Finally, the animals were tested on stepdown passive avoidance under the original drug

This research was supported in part by grants from the San Diego State University Foundation, 263034-326 and 263034-334; and from The National Institute of Mental Health, MH-24329-01, awarded to Sandra Mollenauer and Rod Plotnik. condition. This test provided further information on drug effectiveness and determined whether the present dose of scopolamine had effects comparable to those reported in the literature (Calhoun \& Smith, 1968).

\section{METHOD}

\section{Subjects}

The subjects, purchased from Simenson Co., were 14 male, hooded, Long-Evans rats, weighing $300-400 \mathrm{~g}$ at the beginning of the experiment. The rats were individually housed beginning 2 weeks prior to the start of the experiment. Rats had unlimited access to water and were maintained on a 23-h food-deprivation schedule. They were fed a fixed amount, approximately $15 \mathrm{~g}$, each day after testing. One rat was discarded for failure to satisfy training criteria described below.

\section{Apparatus}

Platform apparatus. This apparatus, $18 \mathrm{~cm}$ wide and $19 \mathrm{~cm}$ high, fit just inside the door of the rat's home cage, completely filling the front of the cage. On the side of the apparatus facing the rat was an enclosed rectangular platform of black Lucite, $31 / 2 \mathrm{~cm}$ high and $51 / 2 \mathrm{~cm}$ deep. In the surface of the platform were two $4-\mathrm{cm}$-diam holes, $6 \mathrm{~cm}$ apart, separated by a clear Plexiglas divider. Two identical, removable, Lucite cups, 4-cm in diameter, were mounted beneath the holes in the platform. The tops of the cups were made of black Lucite perforated with pin holes, which permitted smell but not sight of the cups' contents. For delivery of sucrose pellets onto the surface of the cups, a glass tube, $5 \mathrm{~mm}$ in diameter, protruded through the platform on each side and extended to within several millimeters of the surface of the cup.

Head poke apparatus. The head-poke apparatus was similar to the platform apparatus, with the following differences. A clear Lucite panel, $18 \mathrm{~cm}$ wide and $19 \mathrm{~cm}$ high, was affixed to the front of the platform, blocking access to the food cups except through two small, 3.8-cm-diam, holes in the face of the panel. The two holes were separated by a clear Lucite divider, $11 \mathrm{~cm}$ high, which protruded $5.5 \mathrm{~cm}$ into the rat's cage. The rat's only means of access to the cups and reinforcement was by poking its head through the 3.8-cm holes.

Passive avoidance. The apparatus used to test passive avoid- 
ance was a chamber $29 \times 35 \times 20 \mathrm{~cm}$ high, with a grid floor. It was enclosed in a sound-attenuated, ventilated box. In one corner of the chamber was a wood platform, $15 \times 15 \mathrm{~cm}$, that was raised $4.0 \mathrm{~cm}$ off the grid floor. Scrambled shock could be applied to the grid floor. A switch affixed to the underside of the platform operated a counter whenever the animal stepped off the platform.

\section{Procedure}

Smell discrimination. Rats were taken in their home cages to a test room provided with white noise. Rats were individually given 26 trials each day with approximately 1 min between trials. On each trial the apparatus was placed into the front of the animal's home cage, with one of the cups containing food mash (one part Purina Chow and one part water) and one cup empty. The contents of the cups were not visible to the rat. Each rat was trained to approach the cup containing food within $10 \mathrm{sec}$, the only cue being odor, and remain with its head over the cup for 10 consecutive seconds. After its head had remained over the cup for $10 \mathrm{sec}$, two $45-\mathrm{mg}$ sucrose pellets were manually dispensed to the top of the baited food cup. An animal was permitted to sample both food cups, but was reinforced only if it selected the correct cup within $10 \mathrm{sec}$ of placing the apparatus into the cage and only if its head remained over the selected cup for 10 consecutive seconds. Typically, the rats sampled the cups very quickly; if a rat happened to sample the unbaited cup first, it quickly switched to the other cup. After the rat had been reinforced and allowed to consume the pellets, the apparatus was withdrawn from the cage. The cups were removed, wiped with a cloth to remove any possible saliva, and replaced, with the correct side alternated according to the Gellerman series. The apparatus was then placed back into the home cage for the next trial. On Days 1-4, the animals were allowed $15 \mathrm{sec}$ to approach the apparatus and select one of the cups. One rat, on Day 4, failed to reach this criterion on any of the 20 trials and was discarded. Beginning on Day 5, the animals were required to satisfy the dual criteria of approaching and selecting a cup within $10 \mathrm{sec}$ and remaining over the selected cup for 10 consecutive seconds. By Day 10, all animals had reached the training criterion of $85 \%$ accuracy on 2 consecutive days.

On Day 11, all rats were injected 30 min before testing with physiological saline $(1 \mathrm{ml} / \mathrm{kg}, \mathrm{IP})$ in order to adapt them to the injection procedure.

On Day 12, the rats were randomly assigned to treatments, seven to saline (SAL) and six to scopolamine (SCO). Thirty minutes before testing, they were injected IP with physiological saline in a volume of $1 \mathrm{ml} / \mathrm{kg}$, or with $0.8 \mathrm{mg} / \mathrm{kg}$ scopolamine hydrobromide in the same volume. The experimenter was blind to drug treatment, and data were also recorded independently by a second observer, blind to drug treatment. The procedure was the same as for Day 11, except that $15 \mathrm{sec}$ were permitted for selection of the correct cup. The longer latency was used because it had been observed in pilot work with scopolamine-treated rats that presentation of the apparatus seemed to be a stimulus for exploratory behavior. The time that the rat was required to hover over the correct cup before reinforcement remained the same, 10 consecutive seconds.

Second discrimination test and head poke. Two weeks after the first discrimination test, described above, the rats were trained to perform a smell discrimination response on both platform and head-poke apparatuses. On Day 1, the rats were first given five trials on the platform apparatus followed by 15 trials on the head-poke apparatus. By the end of the 15 trials on Day 1, all rats were locating the correct cup by poking their heads through the hole within $10 \mathrm{sec}$ and hovering over it for $10 \mathrm{sec}$. On Day 2, the rats were given 10 trials on each apparatus, and on Day 3 they were given 20 trials on the platform apparatus, followed by 10 trials on the head poke apparatus. On Day 4, the rats were injected $30 \mathrm{~min}$ before testing using the procedure previously described. Then each rat was first given 20 trials with the platform apparatus immediately followed by 10 trials with the head- poke apparatus. On both apparatuses, criteria for defining a correct response were the same as before; the rat was required to make the correct response within $15 \mathrm{sec}$ and to hover over the correct cup for 10 consecutive seconds.

Passive avoidance. One week later, the rats were tested for step-down passive avoidance under the same drug treatment as for the first discrimination test. Injections were administered as previously described $60 \mathrm{~min}$ before the passive avoidance test. The procedure for passive avoidance was similar to that used previously (Mollenauer et al., 1974). An animal was first placed in the dimly lit apparatus and given a $21 / 2-\min$ habituation period, during which no shock was applied to the grid floor. At the end of this $21 / 2-\min$ period, three additional houselights came on in the chamber simultaneously with a $0.8-\mathrm{mA}$ shock, which was applied to the grid floor continuously for the remainder of the session, $121 / 2 \mathrm{~min}$. During the $121 / 2$-min session, the rat's responses were divided into (1) acquisition: the number of step-down responses recorded before the animal had learned to remain on the platform for 30 consecutive seconds: and (2) avoidance: the number of step-down responses recorded after the animal had remained on the platform for 30 consecutive seconds. Pilot work had shown that normal (untreated) animals that had remained on the platform for 30 consecutive seconds very rarely stepped down during the remainder of the test period.

\section{RESULTS}

\section{Smell Discrimination}

The rat was considered to have made a correct response if it approached the correct cup within $15 \mathrm{sec}$ and then remained or hovered with its head over the cup for 10 consecutive seconds. For all rats, these responses were recorded independently by two observers. The data recorded by these observers were nearly identical, as shown by the reliability coefficient of .99 for errors per rat. The data reported below were from the observer who had served as experimenter from the beginning of this experiment.

Based on mean percent correct on all 20 discrimination trials, the SCO rats showed an impairment of discrimination performance. The mean percent correct for SCO rats $(\mathbf{8 1 . 7 \% )}$ ) was significantly poorer than the mean percent correct for SAL rats $(\mathrm{M}=94.2 \%: \mathrm{t}=2.33, \mathrm{df}=11, \mathrm{p}<.05)$. However, examination of the data showed that most of the errors of the SCO rats occurred during the first half of the test. A comparison based on mean percent correct showed that the SCO rats performed significantly better on the last 10 trials than on the first trials $(M=91.7$ and 71.0, respectively, $t=2.74$, $\mathrm{df}=5, \mathrm{p}<.05$ ); the same comparison for SAL rats showed no significant difference between the last 10 trials and the first 10 trials $(M=92.8$ and 95.7, respectively, $t=-.79$ ).

Additional comparisons based on the first 10 and last 10 trials also confirmed this effect. On the first 10 trials, SCO performance was significantly poorer than SAL performance $(t=2.89, \mathrm{df}=11, \mathrm{p}<.05)$, and it did not differ significantly from chance (50\% correct) $(t=2.4, \mathrm{df}=5)$. In contrast, on the last 10 trials SCO performance did differ significantly from chance $(\mathrm{t}=13.4, \mathrm{df}=5, \mathrm{p}<.001)$ and did 
not differ significantly from SAL $(t=0.21)$. SAL performance differed significantly from chance on both the first 10 trials $(t=22.3, \mathrm{df}=6, \mathrm{p}<.001)$ and the last 10 trials $(t=11.67, \mathrm{df}=6, \mathrm{p}<.001)$.

Two kinds of errors were made by the rats, time errors or choice errors. A time error was recorded if the rat failed to approach the correct cup within the 15 -sec limit or, having done so, failed to remain there for 10 consecutive seconds. A choice error was recorded if the rat selected the incorrect cup and hovered over it for the remaining time. Of the errors made by SCO rats on the first 10 trials, $95 \%$ were time errors. These animals typically explored both cups repeatedly without remaining long enough over either cup to score a correct response or choice error. On the last 10 trials, the pattern was different. Only three of the SCO rats were making errors and they were making very few, of which $80 \%$ were choice errors rather than time. It would probably be a mistake to conclude that there was a real difference in the proportion of choice errors for SCO rats on the first 10 and last 10 trials. Rather, the time errors on early trials probably precluded choice errors; given sufficient time, the rats might have made more choice errors. Of the SAL rats, only three were making errors, and these were making too few to warrant comparison between time and choice.

\section{Second Smell Discrimination and Head Poke}

The results from the second discrimination test are presented in Figure 1. The figure shows mean percent correct responses on blocks of five trials. As the figure shows, these results replicated those of the first discrimination test. The SCO rats showed a significant improvement from the first 10 trials to the last 10 trials $(M=75$ and $95.7 \%$, respectively,

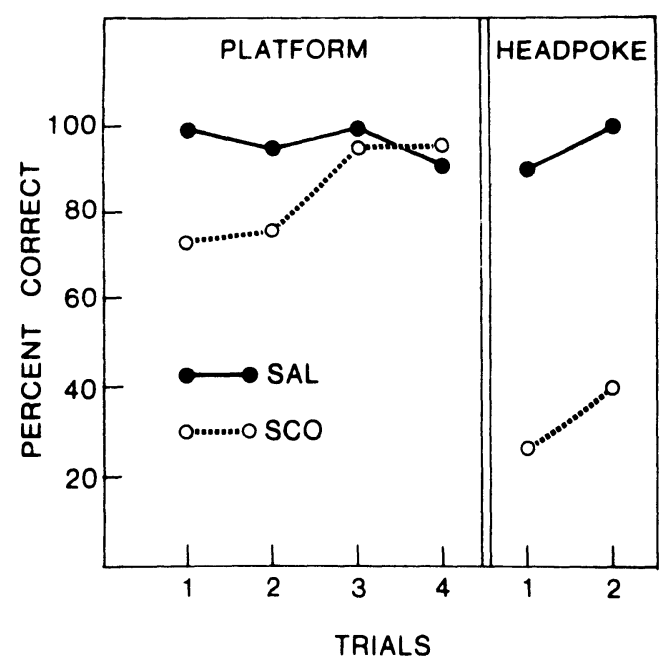

Figure 1. Mean percent correct responses on smell discrimination, tested first with platform apparatus followed by head-poke apparatus. TRIALS = blocks of five trials. $\mathrm{t}=6.48, \mathrm{df}=6, \mathrm{p}<.001)$, but not on the last 10 trials $(t=.34)$. Of the errors made by SCO rats on the first 10 trials, again, the majority $(82 \%)$, were time errors. In fact, very few choice errors were made by any of the rats on this test. Except for the small number of choice errors made by SCO rats on the first 10 trials, there were no choice errors on this test, either for SAL or SCO rats.

The points on the extreme right of Figure 1 show the mean percent correct on the headpoke apparatus. It is clear that the SCO and SAL differences reemerged strongly on the head-poke task $(t=4.73$, $\mathrm{df}=11, \mathrm{p}<.001)$. The SAL rats continued to discriminate with near perfect scores; in contrast, the performance of the SCO rats deteriorated badly. Thus, the SAL performance differed significantly from chance $(t=21.9, \mathrm{df}=5, \mathrm{p}<.001)$, and the SCO performance did not $(t=1.28, \mathrm{df}=6)$.

All but one of the errors made by SCO rats on the head-poke apparatus were time errors. The SCO rats made repeated approaches to the holes without actually putting their heads through; they appeared to withdraw when their vibrissae touched the rim of the hole: As Figure 1 shows, the SAL rats made very few errors on headpoke; all errors by the SAL rats were time errors rather than choice.

\section{Passive Avoidance}

The passive avoidance performance was analyzed separately for acquisition responses, those made before the animal had remained on the platform for 30 consecutive seconds; avoidance responses, those made after the animal had remained on for that period; and total responses, those made during both periods. On total step-down, the SCO rats made significantly more responses than the SAL rats $(M=107.3$ and 37.7, respectively, $t=3.52, \mathrm{df}=11$, $p<.01)$. The SCO rats were also impaired on acquisition. They made an average of 70.2 responses before remaining on the platform for $30 \mathrm{sec}$, as compared to an average of 26.7 for the SAL rats $(t=2.70, \mathrm{df}=11, \mathrm{p}<.05)$. The SCO rats also made more responses during the avoidance period, $\mathrm{M}=37.2$ as compared to 11.0 for $\mathrm{SAL}$, but the difference was not significant $(\mathrm{t}=2.14, \mathrm{df}=11)$.

\section{DISCUSSION}

Overall discrimination performance was significantly impaired by the present dosage of scopolamine, a finding which is consistent with previous research on discrimination tasks and anticholinergic drugs (Whitehouse, 1964). However, most of the errors by SCO rats were on early trials, and $95 \%$ of the errors by SCO rats were time errors in which they failed to approach the cup within the 15-sec limit or failed to remain over it for 10 consecutive seconds. That is, 
SCO rats were not making incorrect choices on early trials; rather, they were failing to make any choices, correct or incorrect. As the SCO rats began to approach the apparatus and execute choices, their choices were correct. During the second half of the test the SCO rats maintained near perfect discrimination performance. Since the rats treated with scopolamine were able to perform the discrimination task using only an odor cue, it seems clear that scopolamine had not rendered the rats anosmic. At the same time, these results would not rule out the possibility of some subtle olfactory impairment.

The present results indicate that one effect of scopolamine is to interfere with the onset of reinforced behavior. The performance of SCO rats was impaired on early trials but showed increased improvement over trials and was virtually identical to that of SAL rats on later trials. This result was similar to a previous finding that scopolamine caused a delay in the onset of food-reinforced bar pressing, but did not affect the rate of response once the animals had begun barpressing (Plotnik, Mollenauer, \& Milberg, in press). The behavior of the scopolaminetreated rats in the barpress situation and in the smell discrimination was consistent with the view that scopolamine-treated rats behave as if they had not been habituated to the apparatus (Carlton, 1969; Feigley \& Hamilton, 1971). Together, these findings suggest the importance of looking separately at early and late performance of scopoloamine-treated animals.

The head-poke and step-down passive avoidance tasks were included to provide additional measures of drug effectiveness. Performance on both tasks was significantly impaired by scopolamine. The fact that passive avoidance was disrupted shows that the dose of scopolamine used in the present research had effects comparable to those used in the literature (Calhoun \& Smith, 1968). This same dose of scopolamine had also been shown to decrease fear or defensive responses of rats to a cat (Plotnik, et al., 1974).

The results of the head-poke test may have implications for another sensory system, the vibrissae. SCO rats that were performing at near perfect levels on the platform apparatus showed a breakdown of performance when tested with the head-poke apparatus. A possible interpretation of this effect is that the drug had altered vibrissae sensibility. Alternatively, the impairment of head-poke performance may have reflected the greater difficulty of the problem, or the greater availability of dis- tracting stimuli. The suggestion that scopolamine might have affected the vibrissae was prompted by observations of the scopolamine-treated rats. The SCO animals frequently approached the correct hole and withdrew as soon as their vibrissae touched the rim of the hole. This observation is consistent with previous research on head-poke. Feigley and Hamilton (1971) reported shorter duration of headpoke responding following scopolamine. They interpreted their results as evidence for impairment of habituation, and suggested a possible role for increased vibrissae sensitivity.

In summary, previous research had raised the possibility that the anticholinergic drug, scopolamine, caused a blockade of olfactory perception. In the present research, the same dose of scopolamine caused a delay in the onset of smell discrimination performance, but did not impair choice behavior. Once scopolamine-treated rats began performing, they showed near-perfect discrimination. These results cannot rule out the possibility of some subtle action of scopolamine on the olfactory system, but they do rule out the possibility of drug-induced anosmia.

\section{REFERENCES}

Calhoun, W. H., \& Smith, A. A. Effects of scopolamine on acquisition of passive avoidance. Psychopharmcologia, 1968, 13. 201-209.

Carlton, P. L. Brain-acetylcholine and inhibition. In J. Tapp (Ed.), Reinforcement and behavior. New York: Academic Press, 1969.

Feigley, D. A., \& Hamilton, L. W. Response to novel environment following septal lesions or cholinergic blockade in rats. Journal of Comparative and Physiological Psychology, 1971. 76, $496-504$.

76, 496-504.

Plotnik, R., Mollenauer, S., \& Milberg, L. Scopolamine and food-reinforced behavior in the rat. Physiological Psychology, in press.

Plotnik, R., Mollenauer, S., \& Snyder, E. Fear reduction in the rat following central cholinergic blockade. Journal of Comparative and Physiological Psychology, 1974, 86, 1074-1082.

Mollenauer, S., Plotnik, R., \& Snyder, E. Effects of olfactory bulb removal on fear responses and passive avoidance in the rat. Physiology and Behavior, 1974, 12, 141-144.

Spector, S. A., \& Hull, E. M. Anosmia and mouse killing by rats: A non-olfactory role for the olfactory bulbs. Journal of Comparative and Physiological Psychology, 1972, 80, 354-356.

Whitehouse, J. M. Effects of atropine on discrimination learning in the rat. Journal of Comparative and Physiological Psychology, 1964, 57, 13-15.

(Received for publication January 6, 1976; revision accepted April 7, 1976.) 